MATHEMATICS OF COMPUTATION

Volume 79, Number 270, April 2010, Pages 967-981

S $0025-5718(09) 02292-3$

Article electronically published on September 8, 2009

\title{
ON THE SPAN OF POLYNOMIALS WITH INTEGER COEFFICIENTS
}

\author{
STEFANO CAPPARELLI, ALBERTO DEL FRA, AND CARLO SCIÒ
}

\begin{abstract}
Following a paper of R. Robinson, we classify all hyperbolic polynomials in one variable with integer coefficients and span less than 4 up to degree 14, and with some additional hypotheses, up to degree 17 . We conjecture that the classification is also complete for degrees 15,16 , and 17 .

Besides improving on the method used by Robinson, we develop new techniques that turn out to be of some interest.

A close inspection of the polynomials thus obtained shows some properties deserving further investigations.
\end{abstract}

\section{INTRODUCTION}

The study of polynomials in one variable and integer coefficients is a topic of remarkable interest that has been investigated by a number of outstanding researchers such as Stieltjes, Kronecker, Chebyshev, Hermite, Schur, and Pólya.

Besides their intrinsic interest in various branches of Mathematics such as, for example, Algebra and Analysis, one should recall their importance in the applications, from Probability Theory to Physics and Engineering.

In many situations, it is important to count the number of real roots of given polynomials in a given domain. A special place is reserved to the classification of monic polynomials with integer coefficients, irreducible (over the integers), that in such a domain have all real roots.

A classic result in this direction is one of Kronecker [3] who determined the infinite family of all such polynomials having roots in the interval $[-2,2]$.

Another remarkable result, due to Schur [9], says that for every interval $I$ with length less than 4 , the set of such polynomials with roots in $I$ is finite.

On the other hand, Robinson, 7], showed that, given any interval of length greater than 4 , there are infinitely many polynomials of the desired type, with roots in the given interval.

It is still open, as far as we can tell, the problem of the finiteness of the number of such polynomials with roots in an interval of length exactly 4, except for those intervals obtained as an integer translation of $[-2,2]$, for which Kronecker's result can easily be extended.

Following a common terminology, we call hyperbolic a polynomial in one variable having all real roots. We call span of such a polynomial the difference between the largest and the smallest root. We recall that irreducible hyperbolic polynomials with integer coefficients necessarily have all distinct roots. From these results, it

Received by the editor November 10, 2008 and, in revised form, April 4, 2009.

2000 Mathematics Subject Classification. Primary 12D10; Secondary 30C15, 11C08.

(C)2009 American Mathematical Society 
is natural to think that the set, which we denote by $\mathcal{P}_{n}$, of the hyperbolic monic irreducible polynomials over the integers whose span is less that 4 deserves a special investigation. We also denote by $\mathcal{K}_{n}$ the set consisting of all those polynomials of $\mathcal{P}_{n}$ all of whose roots lie in $[-2,2]$. Finally we set $\mathcal{N}_{n}$ to be the complement of $\mathcal{K}_{n}$ in $\mathcal{P}_{n}$.

A fundamental work on such polynomials was done by Robinson [8] who classified them, up to equivalence, up to degree 6 and was able to study them up to degree 8 only partially, because of the intrinsic computational complexity of the problem. In the present paper, which we see as an ideal continuation of Robinson's work, we are able to confirm Robinson's list for the degrees 7 and 8 and to extend it up to degree 14, using more modern computing tools and a slightly more refined procedure. Moreover, we obtained a list of polynomials up to degree 17, by imposing some reasonable restrictions inspired by a kind of statistical analysis of such polynomials.

We have observed that the number of polynomials in our list that do not satisfy Kronecker's conditions seems to diminish drastically as the degree $n$ increases.

\section{RoBinson's METHOD}

Let $f_{n}(x)$ be an irreducible monic polynomial in $\mathbf{Z}[x]$ of degree $n>1$, having only real roots:

$$
f_{n}(x)=x^{n}+a_{1} x^{n-1}+a_{2} x^{n-2}+\cdots+a_{n} .
$$

Supposing the roots are listed as follows: $\alpha_{1}<\alpha_{2}<\cdots<\alpha_{n}$, the span of $f_{n}(x)$ equals the difference $\alpha_{n}-\alpha_{1}$.

We say that such a polynomial $f_{n}(x)$ with roots $\alpha_{1}, \ldots, \alpha_{n}$ is equivalent to $g_{n}(x)$ with roots $\beta_{1}, \ldots, \beta_{n}$ if, up to reordering, $\beta_{i}= \pm \alpha_{i}+k$, for $i=1, \ldots, n$ and $k \in \mathbf{Z}$. We can and will choose a representative polynomial in each equivalence class so that the average of the roots is between 0 and $\frac{1}{2}$. In those cases where the average is precisely 0 or $\frac{1}{2}$, then, following Robinson, we agree to choose the polynomial for which the average $\frac{1}{2}\left(\alpha_{n}+\alpha_{1}\right)$ is the closest to $\frac{1}{4}$.

Denote by $f_{n}^{(i)}$ the $i$-th derivative of the polynomial $f_{n}$. The derivative $f_{n}^{(i)}$ is still a hyperbolic polynomial. Let

$$
\alpha_{i, 1}<\alpha_{i, 2}<\cdots<\alpha_{i, n-i}
$$

be the roots of $f_{n}^{(i)}$. Clearly, every root $\alpha_{i, j}$ corresponds to a relative extremum of $f_{n}^{(i-1)}$. Obviously, the value of $f_{n}^{(i-1)}\left(\alpha_{i, j}\right)$ is positive at a relative maximum point, and the value of $f_{n}^{(i-1)}\left(\alpha_{i, j}\right)$ is negative at a relative minimum point.

For the $(n-1)$-th derivative of $f_{n}$ (of degree 1 ), the following is the only root:

$$
\alpha_{n-1,1}=-\frac{a_{1}}{n} \text {. }
$$

The assumption that $0 \leq-\frac{a_{1}}{n} \leq \frac{1}{2}$ implies that

$$
\frac{-n}{2} \leq a_{1} \leq 0
$$

which gives us an upper and lower bound for the first coefficient.

We get an upper bound for $a_{2}$ since $f_{n}^{(n-2)}\left(\alpha_{n-1,1}\right)<0$; hence

$$
0>\frac{f_{n}^{(n-2)}\left(\alpha_{n-1,1}\right)}{(n-2) !}=\frac{n(n-1)}{2} \frac{a_{1}^{2}}{n^{2}}+(n-1) a_{1}\left(\frac{-a_{1}}{n}\right)+a_{2}
$$


i.e.,

$$
a_{2}<\frac{n-1}{2 n} a_{1}^{2} .
$$

We are able to obtain a lower bound for $a_{2}$, which was not given by Robinson. The roots of the second degree polynomial $f_{n}^{(n-2)}$ are

$$
\begin{aligned}
& \alpha_{n-2,1}=\frac{-(n-1) a_{1}-\sqrt{(n-1)^{2} a_{1}^{2}-2 n(n-1) a_{2}}}{n(n-1)}, \\
& \alpha_{n-2,2}=\frac{-(n-1) a_{1}+\sqrt{(n-1)^{2} a_{1}^{2}-2 n(n-1) a_{2}}}{n(n-1)},
\end{aligned}
$$

and the span of the $(n-2)$-th derivative is

$$
\alpha_{n-2,2}-\alpha_{n-2,1}=\frac{2 \sqrt{(n-1)^{2} a_{1}^{2}-2 n(n-1) a_{2}}}{n(n-1)} .
$$

If this span is less than $k$ for some $k$, then

$$
(n-1)^{2} a_{1}^{2}-2 n(n-1) a_{2}<\frac{k^{2}}{4} n^{2}(n-1)^{2},
$$

i.e.,

$$
a_{2}>\frac{n-1}{2 n} a_{1}^{2}-\frac{k^{2}}{4}\left(\begin{array}{l}
n \\
2
\end{array}\right) .
$$

Now, as a simple consequence of the Gauss-Lucas Theorem, the span of the derivative is smaller than the span of $f_{n}$ which, in turn, is assumed to be smaller than 4 , so we certainly have the following lower bound for $a_{2}$ :

$$
a_{2}>\frac{n-1}{2 n} a_{1}^{2}-4\left(\begin{array}{l}
n \\
2
\end{array}\right) \text {. }
$$

We get a sharper lower bound by using the following theorem (see Theorem 6.1.6 of [5]):

Theorem 2.1. Let $D_{n}$ be the span of $f_{n}(n \geq 3)$ and $D_{2}$ the span of $f_{n}^{(n-2)}$. We then have

$$
D_{2} \leq c_{n} D_{n}
$$

where

$$
c_{n}:= \begin{cases}(n-1)^{-1 / 2} & \text { if } n \text { is even } \\ \frac{(n+1)^{1 / 2}}{n} & \text { if } n \text { is odd } .\end{cases}
$$

Since $D_{n}<4$, then $D_{2}<4 c_{n}$. By using (2.3), we get

$$
a_{2}>\frac{n-1}{2 n} a_{1}^{2}-4 c_{n}^{2}\left(\begin{array}{l}
n \\
2
\end{array}\right) \text {. }
$$

Summing up we have proved

Proposition 2.2.

$$
\frac{n-1}{2 n} a_{1}^{2}-4 c_{n}^{2}\left(\begin{array}{l}
n \\
2
\end{array}\right)<a_{2}<\frac{n-1}{2 n} a_{1}^{2}
$$


Once we have determined the coefficients $a_{1}$ and $a_{2}$, we can get upper and lower bounds for $a_{3}$. The fact that $f_{n}^{(n-3)}\left(\alpha_{n-2,1}\right)>0$ implies

$$
\left(\begin{array}{l}
n \\
3
\end{array}\right) \alpha_{n-2,1}^{3}+\left(\begin{array}{c}
n-1 \\
2
\end{array}\right) a_{1} \alpha_{n-2,1}^{2}+\left(\begin{array}{c}
n-2 \\
1
\end{array}\right) a_{2} \alpha_{n-2,1}+a_{3}>0
$$

while $f_{n}^{(n-3)}\left(\alpha_{n-2,2}\right)<0$ implies

$$
\left(\begin{array}{l}
n \\
3
\end{array}\right) \alpha_{n-2,2}^{3}+\left(\begin{array}{c}
n-1 \\
2
\end{array}\right) a_{1} \alpha_{n-2,2}^{2}+\left(\begin{array}{c}
n-2 \\
1
\end{array}\right) a_{2} \alpha_{n-2,2}+a_{3}<0
$$

from which we deduce

Proposition 2.3. If $-\sum_{j=0}^{2}\left(\begin{array}{l}n-j \\ 3-j\end{array}\right) a_{j} \alpha_{n-2,1}^{3-j}<-\sum_{j=0}^{2}\left(\begin{array}{l}n-j \\ 3-j\end{array}\right) a_{j} \alpha_{n-2,2}^{3-j}$, then

$$
-\sum_{j=0}^{2}\left(\begin{array}{c}
n-j \\
3-j
\end{array}\right) a_{j} \alpha_{n-2,1}^{3-j}<a_{3}<-\sum_{j=0}^{2}\left(\begin{array}{c}
n-j \\
3-j
\end{array}\right) a_{j} \alpha_{n-2,2}^{3-j}
$$

Analogously we can determine upper and lower bounds for $a_{4}$.

Proposition 2.4. If $-\sum_{j=0}^{3}\left(\begin{array}{l}n-j \\ 4-j\end{array}\right) a_{j} \alpha_{n-3,2}^{4-j}<-\sum_{j=0}^{3}\left(\begin{array}{l}n-j \\ 4-j\end{array}\right) a_{j} \alpha_{n-3,2 s-1}^{4-j}$, for $s=$ 1,2 , then

$$
-\sum_{j=0}^{3}\left(\begin{array}{l}
n-j \\
4-j
\end{array}\right) a_{j} \alpha_{n-3,2}^{4-j}<a_{4}<-\sum_{j=0}^{3}\left(\begin{array}{l}
n-j \\
4-j
\end{array}\right) a_{j} \alpha_{n-3,2 s-1}^{4-j}
$$

and therefore we get the best upper bound by choosing the minimum of the two values for $s=1,2$.

Remark 2.5. The roots $\alpha_{n-3, t}(t=1,2,3)$ of the polynomial $f_{n}^{(n-3)}$ are known once we have computed $a_{1}, a_{2}, a_{3}$.

In general, we have

Proposition 2.6. If $h$ is even, let, for $r=1,2, \ldots, \frac{h}{2}-1$ and $s=1,2, \ldots, \frac{h}{2}$, $L_{h}=\max _{r}\left[-\sum_{j=0}^{h-1}\left(\begin{array}{c}n-j \\ h-j\end{array}\right) a_{j} \alpha_{n-h+1,2 r}^{h-j}\right], R_{h}=\min _{s}\left[-\sum_{j=0}^{h-1}\left(\begin{array}{l}n-j \\ h-j\end{array}\right) a_{j} \alpha_{n-h+1,2 s-1}^{h-j}\right]$.

If $h$ is odd, let, for $s=1,2, \ldots, \frac{h-1}{2}$, $L_{h}=\max _{s}\left[-\sum_{j=0}^{h-1}\left(\begin{array}{c}n-j \\ h-j\end{array}\right) a_{j} \alpha_{n-h+1,2 s-1}^{h-j}\right], R_{h}=\min _{s}\left[-\sum_{j=0}^{h-1}\left(\begin{array}{l}n-j \\ h-j\end{array}\right) a_{j} \alpha_{n-h+1,2 s}^{h-j}\right]$.
$\quad$ If $L_{h}<R_{h}$, we have $L_{h}<a_{h}<R_{h}$.

If at any step $L_{h} \geq R_{h}$ or the interval $\left[L_{h}, R_{h}\right]$ does not contain integers, the algorithm stops. At the $h$-th step $(h>2)$ we also check whether the span $d_{h}$ is less than 4. 


\section{An application of A theorem of Hermite}

Our method is largely based on Robinson's method combined, in the case of the determination of even index coefficients, with the classic theorem of Hermite [2] (see also [1] and [4]) which allows us to improve the upper bound $R_{h}$.

Theorem 3.1. Given a real polynomial $f_{n}(x)=x^{n}+a_{1} x^{n-1}+a_{2} x^{n-2}+\cdots+a_{n}$, with (complex) roots $\alpha_{1}, \ldots, \alpha_{n}$, consider the matrix

$$
S=\left(\begin{array}{cccc}
s_{0} & s_{1} & \ldots & s_{n} \\
s_{1} & s_{2} & \ldots & s_{n+1} \\
\ldots & \ldots & \ldots & \ldots \\
s_{n} & s_{n+1} & \ldots & s_{2 n-2}
\end{array}\right)
$$

where $s_{j}=\sum_{i=1}^{n} \alpha_{i}^{j} \quad(j=0, \ldots, n)$, the $j$-th Newton's symmetric function on the roots of the polynomial.

The signature of $S$ is equal to the number of real roots of $f_{n}(x)$, and the rank of $S$ is equal to the number of distinct roots.

In particular, we deduce:

Corollary 3.2. $f_{n}(x)$ is a hyperbolic polynomial if and only if the matrix $S$ is positive definite.

It is well known that Newton's symmetric functions on the roots of $f_{n}$ are expressible as polynomials in the coefficients $a_{1}, \ldots, a_{n}$. This implies that all the principal minors of $S$ are also polynomials in the coefficients of $f_{n}$.

The conditions that the principal minors of $S$ must be positive yield an upper bound for the coefficients with even index as in the following example concerning the $2 \times 2$ principal minor of $S$ :

$$
\left|\begin{array}{ll}
s_{0} & s_{1} \\
s_{1} & s_{2}
\end{array}\right|=\left|\begin{array}{cc}
n & -a_{1} \\
-a_{1} & a_{1}^{2}-2 a_{2}
\end{array}\right|=(n-1) a_{1}^{2}-2 n a_{2}>0 .
$$

This is equivalent to the upper bound (2.2). In general, the $k \times k$ principal minor is a function of the first $2 k-2$ coefficients, which we may denote by $\Delta_{k}\left(a_{1}, \ldots, a_{2 k-2}\right)$, linear in $a_{2 k-2}$, which has negative coefficient. Hence $\Delta_{k}>0$ is equivalent to an upper bound $b_{2 k-2}$ for $a_{2 k-2}$ in terms of $a_{1}, \ldots, a_{2 k-3}$, which is often stronger, for small $k$, than the upper bound in Proposition 2.6.

We used this algorithm to compute the list of polynomials up to degree 10 . While we found no significant gain in time with respect to Robinson's original method in our implementation, we believe that it could deserve further study. In the next section we are going to show a different algorithm that proved to be remarkably faster.

\section{The SEQUENCE $\left\{q_{k}\right\}$}

Given the hyperbolic polynomial $f_{n}(x)$ as in (2.1), with roots $\alpha_{1}<\cdots<\alpha_{n}$, we will define the sequence $\left\{q_{k}\right\}, k$ odd, by taking $q_{k}$ as the only real root of the equation

$$
\sum_{i=1}^{n}\left(\alpha_{i}-x\right)^{k}=0
$$

Proposition 4.1. $\lim _{k \rightarrow \infty} q_{k}=\frac{\alpha_{1}+\alpha_{n}}{2}$. 
Proof. Assume $\alpha_{1}<\alpha_{2}<\cdots<\alpha_{j_{k}} \leq q_{k} \leq \alpha_{j_{k}+1}<\cdots<\alpha_{n}$.

We have

$$
\left(q_{k}-\alpha_{1}\right)^{k}+\cdots+\left(q_{k}-\alpha_{j_{k}}\right)^{k}=\left(\alpha_{j_{k}+1}-q_{k}\right)^{k}+\cdots+\left(\alpha_{n}-q_{k}\right)^{k} .
$$

Set

$$
\begin{cases}\left(q_{k}-\alpha_{i}\right)^{k}=\epsilon_{k, i}\left(q_{k}-\alpha_{1}\right)^{k} & \left(i=2, \ldots, j_{k}\right), \\ \left(\alpha_{j}-q_{k}\right)^{k}=\eta_{k, j}\left(\alpha_{n}-q_{k}\right)^{k} & \left(j=j_{k}+1, \ldots, n-1\right) .\end{cases}
$$

Equality (4.1) can be rewritten as

$$
\left(q_{k}-\alpha_{1}\right)^{k}\left(1+\epsilon_{k, 2}+\cdots+\epsilon_{k, j_{k}}\right)=\left(\alpha_{n}-q_{k}\right)^{k}\left(1+\eta_{k, j_{k}+1}+\cdots+\eta_{k, n-1}\right) .
$$

Hence, setting $\sum \epsilon_{k, i}=\epsilon_{k}$ and $\sum \eta_{k, j}=\eta_{k}$,

$$
\left(q_{k}-\alpha_{1}\right)^{k}\left(1+\epsilon_{k}\right)=\left(\alpha_{n}-q_{k}\right)^{k}\left(1+\eta_{k}\right)
$$

and so, if we set

$$
\zeta_{k}=\sqrt[k]{\frac{1+\eta_{k}}{1+\epsilon_{k}}}
$$

we have

$$
q_{k}=\frac{\alpha_{1}+\alpha_{n} \zeta_{k}}{1+\zeta_{k}}
$$

To conclude it is enough to show that $\lim _{k \rightarrow \infty} \zeta_{k}=1$. This a consequence of the following two remarks:

i) $\epsilon_{k, i}<\epsilon_{k, i}^{\prime}:=\left(\frac{\alpha_{n}-\alpha_{i}}{\alpha_{n}-\alpha_{1}}\right)^{k} \quad\left(i=2, \ldots, j_{k}\right)$,

$$
\eta_{k, i}<\eta_{k, i}^{\prime}:=\left(\frac{\alpha_{i}-\alpha_{1}}{\alpha_{n}-\alpha_{1}}\right)^{k} \quad\left(i=j_{k}+1, \ldots, n-1\right),
$$

ii) $\epsilon_{k, i}^{\prime} \leq \epsilon_{k, 2}^{\prime} \quad\left(i=2, \ldots, j_{k}\right), \quad \eta_{k, i}^{\prime} \leq \eta_{k, n-1}^{\prime} \quad\left(i=j_{k}+1, \ldots, n-1\right)$.

Using i) and ii) one can show that

$$
\epsilon_{k}<(n-2) \epsilon_{k, 2}^{\prime}, \quad \eta_{k}<(n-2) \eta_{k, n-1}^{\prime} .
$$

From (4.4) it follows that

$$
\frac{1}{1+(n-2) \epsilon_{k, 2}^{\prime}} \leq \frac{1+\eta_{k}}{1+\epsilon_{k}} \leq 1+(n-2) \eta_{k, n-1}^{\prime} .
$$

Since $\frac{\alpha_{n}-\alpha_{i}}{\alpha_{n}-\alpha_{1}}<1$ and $\frac{\alpha_{i}-\alpha_{1}}{\alpha_{n}-\alpha_{1}}<1$, both $\epsilon_{k, n-1}^{\prime}$ and $\eta_{k, 2}^{\prime}$ are infinitesimal. Therefore as $k$ approaches infinity, the first and third member of (4.5) approach 1 and so $\lim _{k \rightarrow \infty} \zeta_{k}=1$.

Remark 4.2. The proof can easily be modified to show the same result even when the roots of $f(x)$ are not all distinct.

Definition 4.3. Let $\mathcal{F}\left(\alpha_{1}, \alpha_{n}\right)$ be the family of all the degree $n$ hyperbolic polynomials with fixed smallest root $\alpha_{1}$ and largest root $\alpha_{n}$.

From Proposition 4.1 it follows that for every polynomial in $\mathcal{F}\left(\alpha_{1}, \alpha_{n}\right)$ the related sequence $\left\{q_{k}\right\}$ approaches the same value $\frac{\alpha_{1}+\alpha_{n}}{2}$.

For any polynomial of the family $\mathcal{F}\left(\alpha_{1}, \alpha_{n}\right)$ we want to estimate the absolute value of the difference $\left|q_{k}-\frac{\alpha_{1}+\alpha_{n}}{2}\right|$. 
One can easily compute that

$$
\left|q_{k}-\frac{\alpha_{1}+\alpha_{n}}{2}\right|=\frac{\left|\left(\zeta_{k}-1\right)\left(\alpha_{n}-\alpha_{1}\right)\right|}{2\left(1+\zeta_{k}\right)} .
$$

We also note that for the particular polynomial $p(x)=\left(x-\alpha_{1}\right)\left(x-\alpha_{n}\right)^{n-1} \in$ $\mathcal{F}\left(\alpha_{1}, \alpha_{n}\right)$ the corresponding value of $\zeta_{k}$ is $\sqrt[k]{n-1}$ and, analogously, for $P(x)=$ $\left(x-\alpha_{1}\right)^{n-1}\left(x-\alpha_{n}\right) \in \mathcal{F}\left(\alpha_{1}, \alpha_{n}\right)$ the corresponding value of $\zeta_{k}$ is $\frac{1}{\sqrt[k]{n-1}}$. Moreover, if we denote by $\bar{q}_{k}$ the generic element of the sequence corresponding to either $p(x)$ or $P(x)$, we have, in both cases,

$$
\left|\bar{q}_{k}-\frac{\alpha_{1}+\alpha_{n}}{2}\right|=\frac{\left|(\sqrt[k]{n-1}-1)\left(\alpha_{n}-\alpha_{1}\right)\right|}{2(\sqrt[k]{n-1}+1)} .
$$

Lemma 4.4. For every polynomial in $\mathcal{F}\left(\alpha_{1}, \alpha_{n}\right)$, for the corresponding value of $\zeta_{k}$, the following inequalities hold: $\frac{1}{\sqrt[k]{n-1}} \leq \zeta_{k} \leq \sqrt[k]{n-1}$

Proof. $\zeta_{k} \leq \sqrt[k]{n-1} \Longleftrightarrow \sqrt[k]{\frac{1+\eta_{k}}{1+\epsilon_{k}}} \leq \sqrt[k]{n-1} \Longleftrightarrow\left(1+\eta_{k}\right) \leq\left(1+\epsilon_{k}\right)(n-1)$. Since $\eta_{k, i} \leq 1$, for all $i$, we have $1+\eta_{k}=1+\eta_{k, j_{k}+1}+\cdots+\eta_{k, n-1} \leq n-j_{k} \leq n-1 \leq$ $\left(1+\epsilon_{k}\right)(n-1)$.

On the other hand, and in the same manner, we can show that $\frac{1}{\sqrt[k]{n-1}} \leq \zeta_{k}$ because $\left(1+\epsilon_{k}\right) \leq\left(1+\eta_{k}\right)(n-1)$.

Proposition 4.5. For every polynomial $f \in \mathcal{F}\left(\alpha_{1}, \alpha_{n}\right)$ we have

$$
\frac{\left|\left(\zeta_{k}-1\right)\left(\alpha_{n}-\alpha_{1}\right)\right|}{2\left(1+\zeta_{k}\right)} \leq \frac{\left|(\sqrt[k]{n-1}-1)\left(\alpha_{n}-\alpha_{1}\right)\right|}{2(\sqrt[k]{n-1}+1)} .
$$

Proof. We distinguish two cases. In the case when $\zeta_{k}>1$ it is enough to check that

$$
(\sqrt[k]{n-1}+1)\left(\zeta_{k}-1\right) \leq(\sqrt[k]{n-1}-1)\left(\zeta_{k}+1\right)
$$

which is equivalent to

$$
\zeta_{k} \leq \sqrt[k]{n-1}
$$

which holds because of Lemma 4.4

In the case when $\zeta_{k}<1$ we can see that

$$
(\sqrt[k]{n-1}+1)\left(1-\zeta_{k}\right) \leq(\sqrt[k]{n-1}-1)\left(\zeta_{k}+1\right)
$$

which is equivalent to

$$
\zeta_{k} \geq \frac{1}{\sqrt[k]{n-1}}
$$

which holds, again, because of Lemma 4.4

The function $m_{k}(f, q):=\sum_{i=1}^{n}\left(\alpha_{i}-q\right)^{k}$ depends on $a_{1}, \ldots, a_{k}, q$, linearly in the $k$-th coefficient $a_{k}$ of the hyperbolic polynomial $f(x)$. So we may also explicitly indicate it by $m_{k}\left(a_{1}, \ldots, a_{k}, q\right)$.

In the recursive procedure to find our polynomials, we will exploit this fact in order to obtain, once the coefficients $a_{1}, \ldots, a_{k-1}$ are given, a lower and an upper bound for $a_{k}$, using two inequalities of the type:

$$
\sum_{i=0}^{k} c_{i}^{\prime} m_{i}\left(a_{1}, \ldots, a_{k}, q\right) \leq 0, \quad \sum_{i=0}^{k} c_{i}^{\prime \prime} m_{i}\left(a_{1}, \ldots, a_{k}, q\right) \geq 0 .
$$


To do this, let us denote, for any degree $k \geq 0$, by $T_{k}(x)$ the $k$-th Chebyshev Polynomial of the first kind. Recall that $T_{0}(x)=1, T_{1}(x)=x$, and $T_{k}(x)=$ $2 x T_{k-1}(x)-T_{k-2}(x), k \geq 2$, and that $T_{k}(x)$ has $k$ real roots in the interval $[-1,1]$ given by the explicit formulas $x_{i}=\cos \left(\frac{\pi}{2} \frac{2 i-1}{k}\right)$. Moreover, the extrema of $T_{k}$ are all \pm 1 and $T_{k}(1)=1$ while $T_{k}(-1)=(-1)^{k}$. We can change the variable in order to consider the Chebyshev's polynomials appropriate to any given interval $[a, b]$ (see [6], 1.3.2). In particular, we consider $T_{k, h}(x)=T_{k}\left(\frac{x}{h}\right)$, which are the Chebyshev polynomials for the interval $[-h, h]$. We shall use

$$
S_{k, h}^{\prime}(x)=T_{k, h}(x)-1, \quad S_{k, h}^{\prime \prime}(x)=T_{k, h}(x)+1 .
$$

We shall need the following easily checked properties of these polynomials: $S_{k, h}^{\prime}(x)$ $\leq 0$ in $[-h, h]$ with maxima equal to 0 and minima equal to $-2 ; S_{k, h}^{\prime \prime}(x) \geq 0$ in $[-h, h]$ with maxima equal to 2 and minima equal to 0 .

Set $S_{k, h}^{\prime}(x)=\sum_{i=0}^{k} c_{i}^{\prime} x^{i}$ and $S_{k, h}^{\prime \prime}(x)=\sum_{i=0}^{k} c_{i}^{\prime \prime} x^{i}$.

For any value $q$ such that $\left|\alpha_{j}-q\right| \leq h$, for all $\alpha_{j}$, we have $\sum_{i=0}^{k} c_{i}^{\prime}\left(\alpha_{j}-q\right)^{i} \leq 0$ and $\sum_{i=0}^{k} c_{i}^{\prime \prime}\left(\alpha_{j}-q\right)^{i} \geq 0$ for all $\alpha_{j}$; hence

$$
\sum_{j=1}^{n} \sum_{i=0}^{k} c_{i}^{\prime}\left(\alpha_{j}-q\right)^{i} \leq 0, \quad \sum_{j=1}^{n} \sum_{i=0}^{k} c_{i}^{\prime \prime}\left(\alpha_{j}-q\right)^{i} \geq 0
$$

or

$$
\sum_{i=0}^{k} c_{i}^{\prime} \sum_{j=1}^{n}\left(\alpha_{j}-q\right)^{i} \leq 0, \quad \sum_{i=0}^{k} c_{i}^{\prime \prime} \sum_{j=1}^{n}\left(\alpha_{j}-q\right)^{i} \geq 0
$$

i.e.,

$$
\sum_{i=0}^{k} c_{i}^{\prime} m_{i}\left(a_{1}, \ldots, a_{k}, q\right) \leq 0, \quad \sum_{i=0}^{k} c_{i}^{\prime \prime} m_{i}\left(a_{1}, \ldots, a_{k}, q\right) \geq 0
$$

These last two inequalities imply, because of the linearity in $a_{k}$, conditions such as

$$
g^{\prime}\left(a_{1}, \ldots, a_{k-1}, q\right) \leq a_{k} \leq g^{\prime \prime}\left(a_{1}, \ldots, a_{k-1}, q\right)
$$

provided $g^{\prime}\left(a_{1}, \ldots, a_{k-1}, q\right) \leq g^{\prime \prime}\left(a_{1}, \ldots, a_{k-1}, q\right)$.

A priori, we do not know the roots $\alpha_{1}, \ldots, \alpha_{n}$. We know, however, that $\lim q_{i}=$ $\bar{q}:=\frac{\alpha_{1}+\alpha_{n}}{2}$ and that

$$
\left|q_{i}-\bar{q}\right| \leq \frac{(\sqrt[i]{n-1}-1)\left(\alpha_{n}-\alpha_{1}\right)}{2(\sqrt[i]{n-1}+1)}<2 \frac{\sqrt[i]{n-1}-1}{\sqrt[i]{n-1}+1}:=\rho_{i}
$$

since $\alpha_{n}-\alpha_{1}<4$, by hypothesis.

If $k$ is even, and assuming we have already determined the coefficients $a_{1}, \ldots$, $a_{k-1}$, then $q_{k-1}$ is determined. Its distance from $\bar{q}$ is less than $\rho_{k-1}$. Therefore, by choosing $q=q_{k-1}$ and $h=2+\rho_{k-1}$ the conditions are satisfied to apply (4.7). If $k$ is odd, an analogous result can be obtained by choosing $q=q_{k-2}$.

Summarizing, the algorithm is as follows.

Given $n, a_{1}, a_{2}$ and $q_{1}=-a_{1} / n$, we consider $m_{3}\left(a_{1}, a_{2}, a_{3}, q_{1}\right)$. Therefore the only variable left is $a_{3}$. We consider the polynomials $S_{3,2+\rho_{1}}^{\prime}(x), S_{3,2+\rho_{1}}^{\prime \prime}(x)$ related to $T_{3,2+\rho_{1}}(x)$. We can now search for integer solutions of inequalities (4.6) with 
$k=3$. If there are no such solutions the algorithm stops. Otherwise, for any solution $a_{3}$, we solve the equation

$$
m_{3}\left(a_{1}, a_{2}, a_{3}, x\right)=0
$$

to find $q_{3}$.

Now we consider $m_{4}\left(a_{1}, a_{2}, a_{3}, a_{4}, q_{3}\right)$, where the only variable is $a_{4}$. We consider the polynomials $S_{4,2+\rho_{3}}^{\prime}(x), S_{4,2+\rho_{3}}^{\prime \prime}(x)$ related to $T_{4,2+\rho_{3}}(x)$. If we do not find integer solutions $a_{4}$ for inequalities (4.6) with $k=4$, we stop. Otherwise we continue by considering, for any such $a_{4}$, the function $m_{5}\left(a_{1}, a_{2}, a_{3}, a_{4}, a_{5}, q_{3}\right)$ (the same value $q_{3}$ is used both for $m_{4}$ and $m_{5}$ ), and so on.

We used this method to compute polynomials up to degree 14. In the following table we compare it with Robinson's by giving the time, in seconds, the two algorithms took to compute the lists for $n=8$ and $n=9$ on the same 9 Gflop processors.

\begin{tabular}{c|r|c|}
$n$ & Robinson & sequence $\left\{q_{k}\right\}$ \\
\hline 8 & $49644 \mathrm{sec}$ & $1143 \mathrm{sec}$ \\
\hline 9 & $1203686 \mathrm{sec}$ & $4943 \mathrm{sec}$ \\
\hline
\end{tabular}

\section{Strengthening of the Bounds}

For degree 15 and higher both the methods of Sections 3 and 4 take an exceedingly long time to complete. So we made some reasonable assumptions, based on a number of empirical observations described below. For degree 15 we used some combinations of these assumptions, always obtaining the same list of polynomials. For degrees 16 and 17 instead we used the method of Section 5.5.

\subsection{Bounds on the spans. Let}

$$
\begin{gathered}
\mathcal{P}_{n}=\left\{f_{n} \mid \operatorname{span}\left(f_{n}\right)<4\right\} \\
\mathcal{P}_{n}^{(i)}=\left\{f_{n}^{(i)} \mid f_{n} \in \mathcal{P}_{n}\right\}, i=0, \ldots, n-2, \\
S_{n}^{i}=\left\{\operatorname{span} f_{n}^{(i)} \mid f_{n}^{(i)} \in \mathcal{P}_{n}^{(i)}\right\} k_{n, i}=\max S_{n}^{i}
\end{gathered}
$$

We tabulated $k_{n, i}$ as a function of $n$ by comparing the span of $f_{n}^{(n-i)}$ with the span of $f_{m}^{(m-i)}$ whenever this is possible, i.e., when $2 \leq i \leq \min (n, m)$.

\begin{tabular}{|l|l|l|l|l|l|l|l|l|l|l|l|l|}
$n$ & $k_{n, n-2} \mid k_{n, n-3}$ & $k_{n, n-4}$ & $k_{n, n-5}$ & $k_{n, n-6}$ & $k_{n, n-7}$ & $k_{n, n-8}$ & $k_{n, n-9}$ & $k_{n, n-10}$ & $k_{n, n-11}$ & $k_{n, n-12}$ \\
\hline 3 & 1.7638 & & & & & & & & & & \\
\hline 4 & 1.9149 & 3.3166 & & & & & & & & & \\
\hline 5 & 1.5492 & 2.6833 & 3.5103 & & & & & & & & \\
\hline 6 & 1.3663 & 2.3664 & 3.1069 & 3.6498 & & & & & & & \\
\hline 7 & 1.2344 & 2.1391 & 2.8401 & 3.3827 & 3.7763 & & & & & & \\
\hline 8 & 1.1339 & 1.9637 & 2.6159 & 3.1351 & 3.5387 & 3.8303 & & & & & \\
\hline 9 & 1.0541 & 1.8257 & 2.4355 & 2.9267 & 3.3187 & 3.6195 & 3.8395 & & & & \\
\hline 10 & 1.0000 & 1.7321 & 2.3131 & 2.7857 & 3.1703 & 3.4778 & 3.7175 & 3.9057 & & & \\
\hline 11 & 0.9271 & 1.6057 & 2.1479 & 2.5949 & 2.9673 & 3.2766 & 3.5303 & 3.7320 & 3.8825 & & \\
\hline 12 & 0.8876 & 1.5374 & 2.0569 & 2.4857 & 2.8436 & 3.1422 & 3.3899 & 3.5985 & 3.7729 & 3.8952 & \\
\hline 13 & 0.8356 & 1.4473 & 1.9392 & 2.3500 & 2.6989 & 2.9968 & 3.2509 & 3.4658 & 3.6446 & 3.7893 & 3.9146 \\
\hline
\end{tabular}


Remark 5.1. Notice that in this table,

$$
k_{n, n-i}>k_{n+1, n+1-i}>\cdots .
$$

If we assume that such an inequality holds in general, we could exploit it for $a_{2}$, by choosing a value of $k$ smaller than $c_{n}$ (see Theorem 2.1) in formula (2.3), by estimating $k_{n, n-2}$ to be smaller than $k_{n-1, n-3}$, which was obtained in the classification of the polynomials of degree $n-1$.

For the other coefficients, one could assume that the span of the $(n-i)$-th derivative is less than $k_{n-1, n-1-i}$, obtained in the classification of polynomials of the previous degree.

5.2. Further restrictions on the spans. For all the polynomials that we obtained up to degree 14 , we computed $\frac{S_{n}^{n-i}}{S_{n}^{n-i+1}}$, for $n=4, \ldots, 14$, the ratio of the span of $f_{n}^{(n-i)}$ over the span of $f_{n}^{(n-i+1)}$ and we took the minimum $\underline{R}_{n}^{i}$ and the maximum $\bar{R}_{n}^{i}$ of such ratios.

We observed that the intervals thus obtained are quite small; moreover, there is a definite stabilizing trend in the sequences $\underline{R}_{3}^{i}, \underline{R}_{4}^{i}, \underline{R}_{5}^{i}, \ldots, \bar{R}_{3}^{i}, \bar{R}_{4}^{i}, \bar{R}_{5}^{i}, \ldots$

In the table below we show the resulting intervals for $i=3,4,5,6$.

\begin{tabular}{|c|c|c|c|c|}
\hline & $\left(\underline{R}_{n}^{3}, \bar{R}_{n}^{3}\right)$ & $\left(\underline{R}_{n}^{4}, \bar{R}_{n}^{4}\right)$ & $\left(\underline{R}_{n}^{5}, \bar{R}_{n}^{5}\right)$ & $\left(\underline{R}_{n}^{6}, \bar{R}_{n}^{6}\right)$ \\
\hline 4 & $(1.71339,1.73205$ & $(1.18598,1.36603$ & & \\
\hline$\overline{5}$ & $(1.72679,1.73205$ & $(1.29707,1.34765$ & $(1.12377,1.22365)$ & \\
\hline$\overline{6}$ & $(1.72950,1.73205$ & $(1.31292,1.34367$ & $(1.15427,1.21707)$ & $(1.07876,1.15923)$ \\
\hline$\overline{7}$ & $(1.73141,1.73205$ & $(1.32581,1.33813$ & $(1.18672,1.20868)$ & $(1.11519,1.14395)$ \\
\hline$\overline{8}$ & $(1.73117,1.73205$ & $(1.33026,1.34267$ & $(1.19500,1.21622)$ & $(1.12471,1.15417)$ \\
\hline 9 & $(1.73108,1.73205$ & $(1.33239,1.33943$ & $(1.19917,1.21107)$ & $(1.13077,1.14674)$ \\
\hline 10 & $(1.73152,1.73205$ & $(1.33464,1.34140)$ & $(1.20293,1.21425)$ & $(1.13625,1.15138)$ \\
\hline$\overline{11}$ & $(1.73165,1.73205$ & $(1.33763,1.34046)$ & $(1.20812,1.21274)$ & $(1.14349,1.14945)$ \\
\hline$\overline{12}$ & $(1.73183,1.73205$ & $(1.33787,1.34267$ & $(1.20848,1.21622)$ & $(1.14399,1.15413)$ \\
\hline & $(1.73200,1.732$ & $(1.33990,1.34176$ & $(1.21183,1.2148$ & $44,1.15233)$ \\
\hline
\end{tabular}

5.3. Restrictions on the location of roots. Given a hyperbolic polynomial $f$ of degree $n$, with (real) roots $x_{1}, \ldots, x_{n}$, let $\mu=\sum_{i=1}^{n} x_{i}$ and $m_{k}=\sum_{i=1}^{n}\left(x_{i}-\mu\right)^{k}$ be, respectively, the average and the Newton functions of the $x_{i}$ 's around $\mu$.

It is well known that the value $c_{3}=m_{3}^{2} / m_{2}^{3}$ gives information about the symmetry of the $x_{i}$ 's around the average $\mu$, while $c_{4}=m_{4} / m_{2}^{2}$ is related to the density of the $x_{i}$ 's around $\mu$.

We have found it useful also to define the following functions, generalizing the above for any integer $k \geq 1$ :

$$
c_{2 k+1}=\frac{m_{2 k+1}^{2 k}}{m_{2 k}^{2 k+1}}, \quad c_{2 k+2}=\frac{m_{2 k+2}^{k}}{m_{2 k}^{k+1}} .
$$

We observed that the values of these functions on the polynomials we determined up to degree 14 vary in quite short intervals, as the following tables show: 


\begin{tabular}{|c|c|c|c|c|c|c|}
\hline & $\left(c_{3}^{\prime}, c_{3}^{\prime \prime}\right)$ & $\left(c_{5}^{\prime}, c_{5}^{\prime \prime}\right)$ & $\left(c_{7}^{\prime}, c_{7}^{\prime \prime}\right)$ & $\left(c_{9}^{\prime}, c_{9}^{\prime \prime}\right)$ & $\left(c_{11}^{\prime}, c_{11}^{\prime \prime}\right)$ & $\left(c_{13}^{\prime}, c_{13}^{\prime \prime}\right)$ \\
\hline 4 & $(0,0.2427)$ & & & & & \\
\hline$F$ & $(0,0.1209)$ & $(0,0.1795)$ & & & & \\
\hline$\overline{6}$ & $(0,0.0841)$ & $(0,0.0545)$ & & & & \\
\hline$\overline{7}$ & $(0,0.0279)$ & $(0,0.0142)$ & $(0,0.0211)$ & & & \\
\hline$\overline{8}$ & $(0,0.0472)$ & $(0,0.0285)$ & $(0,0.0369)$ & & & \\
\hline Y & $(0,0.0619)$ & $(0,0.0397)$ & $(0,0.0443)$ & $(0,0.0621)$ & & \\
\hline$\overline{10}$ & $(0,0.0392)$ & $(0,0.0195)$ & $(0,0.0223)$ & $(0,0.0375)$ & & \\
\hline$\overline{11}$ & $(0,0.0340)$ & $(0,0.0102)$ & $(0,0.0054)$ & $(0,0.0039)$ & $(0,0.0038)$ & \\
\hline 1 & $\overline{(0,0.0213)}$ & $(0,0.0040)$ & $(0,0.0015)$ & $(0,0.0009)$ & $(0,0.0014)$ & \\
\hline$\overline{13}$ & $(0,0.0026)$ & $(0,0.0001)$ & $(0,0)$ & $(0,0)$ & $(0,0)$ & $(0,0)$ \\
\hline
\end{tabular}

\begin{tabular}{|c|c|c|c|c|c|}
$n$ & $\left(c_{4}^{\prime}, c_{4}^{\prime \prime}\right)$ & $\left(c_{6}^{\prime}, c_{6}^{\prime \prime}\right)$ & $\left(c_{8}^{\prime}, c_{8}^{\prime \prime}\right)$ & $\left(c_{10}^{\prime}, c_{10}^{\prime \prime}\right)$ & $\left(c_{12}^{\prime}, c_{12}^{\prime \prime}\right)$ \\
\hline 4 & $(1.1653,1.8457)$ & & & & \\
\hline 5 & $(1.3367,1.7968)$ & & & & \\
\hline 6 & $(1.2857,1.7460)$ & $(1.5873,2.4041)$ & & & \\
\hline 7 & $(1.3858,1.6338)$ & $(1.6855,2.1910)$ & & & \\
\hline 8 & $(1.3910,1.7143)$ & $(1.6823,2.3234)$ & $(1.9454,2.9186)$ & & \\
\hline 9 & $(1.3826,1.6010)$ & $(1.6653,2.0180)$ & $(1.8985,2.5117)$ & & \\
\hline 10 & $(1.3636,1.6314)$ & $(1.6750,2.0647)$ & $(1.8901,2.5278)$ & $(2.0698,3.0006)$ & \\
\hline 11 & $(1.4305,1.5585)$ & $(1.7529,1.9158)$ & $(2.0025,2.2945)$ & $(2.2076,2.6926)$ & \\
\hline 12 & $(1.3846,1.6364)$ & $(1.7094,2.1319)$ & $(1.9789,2.5177)$ & $(2.2102,2.8058)$ & $(2.3739,3.0692)$ \\
\hline 13 & $(1.4477,1.5606)$ & $(1.7716,1.9740)$ & $(2.0497,2.2906)$ & $(2.2922,2.5436)$ & $(2.5053,2.7613)$ \\
\hline
\end{tabular}

where $c_{i}^{\prime}=\min c_{i}, c_{i}^{\prime \prime}=\max c_{i}$ and the minimum and the maximum are computed, up to the fourth decimal digit, over all the polynomials of a given degree we determined.

These tables suggested to us some reasonable restrictions on the values of these functions for the polynomials of degree 15 and up.

Recalling that each $c_{k}$ is a polynomial expression in the first $k$ coefficients $a_{1}, a_{2}, \ldots, a_{k}$ of $f_{n}(x)$, we recursively get a restriction on the coefficient $a_{k}$.

5.4. Restrictions on the range of the coefficients, using Hermite's theorem. We have noticed that for all the polynomials we determined up to degree 14, the actual value of the coefficient $a_{2}$ lies remarkably between $b_{2}-n-1$ and $b_{2}-n+1$ ( $n$ the degree of the polynomial), where $b_{2 i}$ ( $i$ a positive integer) is the bound due to Hermite's Theorem (see Section 3). Similar conditions appear also for the other coefficients of even index of the polynomials: for $i>1, a_{2 i}$ lies between $b_{2 i}-\frac{n}{i}-2$ and $b_{2 i}-\frac{n}{i}+2$. Therefore it seemed natural to impose an analogous restriction to the coefficients of even index. We have no such restriction on the coefficients of odd index.

The range of the coefficients of odd index implied by Proposition 2.6 is far too large; however, we empirically observed that in all the polynomials we have determined up to degree 14, the coefficients of odd index are always quite close to the center of the interval of variability. More precisely, the coefficients are always within twelve units of the center.

5.5. Restrictions on the range of the coefficients, using the method of the sequence $\left\{q_{k}\right\}$. In Section 4 , in order to obtain a range of variability of every coefficient $a_{k}$, we used the $k$-th Chebyshev polynomial of the first kind related to the 
interval $\left[-2-\rho_{k-1}, 2+\rho_{k-1}\right]$. By selecting the Chebyshev polynomials for narrower intervals, one can obviously get a smaller range of variability for the coefficients $a_{k}$. It turned out that all polynomials we determined using the wider intervals would have also been found if we had used the interval $[-2,2]$. Thus, for degrees higher than 14 we decided to use the interval $[-2,2]$, for every $k \geq 3$, in order to remain within a manageable computation. This method turned out to give us results up to degree 17. Of course, the list of polynomials of degree 15 to 17 may not be complete.

\section{Conclusions}

The number of inequivalent polynomials in any given degree that do not belong to Kronecker's family seems to follow a decreasing trend as the degree $n$ increases. Recalling the sets $\mathcal{P}_{n}$ and $\mathcal{N}_{n}$ defined in Section [1, and denoting by $\left|\mathcal{P}_{n}\right|$ and $\left|\mathcal{N}_{n}\right|$ their respective cardinalities, we have the following table.

\begin{tabular}{l|l|r|r|r|r|r|r|r|r|r|r|r|r|r|r|r|}
$n$ & 2 & 3 & 4 & 5 & 6 & 7 & 8 & 9 & 10 & 11 & 12 & 13 & 14 & 15 & 16 & 17 \\
\hline$\left|\mathcal{P}_{n}\right|$ & 4 & 5 & 14 & 15 & 17 & 15 & 26 & 21 & 18 & 11 & 16 & 4 & 10 & 7 & 9 & 3 \\
\hline$\left|\mathcal{N}_{n}\right|$ & 1 & 3 & 10 & 14 & 13 & 15 & 19 & 19 & 15 & 10 & 9 & 4 & 9 & 6 & 3 & 3 \\
\hline
\end{tabular}

Some natural questions present themselves:

i) Is there a degree $n$ for which $\mathcal{N}_{n}$ is empty?

ii) Are there infinitely many such $n$ ?

iii) Is the union of all sets $\mathcal{N}_{n}$ a finite set?

Moreover:

j) Is condition (5.1) true in general?

jj) Theorem 6.1 .2 of $[5]$ implies $\frac{S_{n}^{n-i}}{S_{n}^{n-i+1}} \leq \sqrt{\frac{i}{i-2}}$. In the table of Section 5.2 we observe that for $i=3$ this upper bound is reached, while for $i>3$, the maximum $\bar{R}_{n}^{i}$ stays well below $\sqrt{\frac{i}{i-2}}$. In the same table we also listed $\underline{R}_{n}^{i}$ for which we do not know an analogous lower bound for $i>3$. For $i=3$, Theorem 6.1.6 of [5] gives the lower bound of 1.5, which turns out to be definitely smaller than the observed lower bounds $\underline{R}_{n}^{3}$.

jjj) The first table of Section 5.3 seems to suggest a high level of symmetry for the roots of the polynomial around the average. The second table also shows some regularities that deserve further study. We believe that this could lead to a better understanding of the distribution of the roots.

jv) The most striking observation, in our opinion, concerns the constraints on the coefficients given in Section 5.4 .

$$
b_{2 i}-\frac{n}{i}-2 \leq a_{2 i} \leq b_{2 i}-\frac{n}{i}+2,
$$

where $b_{2 i}$ is determined by Hermite's Theorem. Is this true in general? 


\section{List OF POLYNOMials}

Here we only give the list of the polynomials belonging to $\mathcal{P}_{n}, 9 \leq n \leq 17$, since the list for $n \leq 8$ is given by Robinson in 7 . We also give the span of every polynomial and point out if it belongs to $\mathcal{K}_{n}$. We recall that the list of polynomials for degrees 15, 16 and 17 might not be complete.

\begin{tabular}{|c|c|c|c|c|c|c|c|c|c|c|c|c|c|c|c|c|c|c|}
\hline$\left.{ }^{17}\right|_{x}$ & 1 & & & & 12 & 1 & $r^{10}$ & $\mid x^{9}$ & $x^{\varepsilon}$ & 7 & $x^{6}$ & $x^{5}$ & ${ }^{5} \quad x^{4}$ & $x^{3}$ & $x^{2}$ & $x^{1}$ & $x^{0}$ & an \\
\hline 0 & & 0 & & 0 & 0 & 0 & 0 & & - & & 20 & -3 & $3-33$ & 6 & 18 & -1 & -1 & 3.99871 \\
\hline - & & 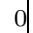 & 0 & 0 & 0 & 0 & 0 & 1 & -4 & -2 & 20 & -3 & $3-33$ & 6 & 19 & -1 & -2 & 540 \\
\hline 0 & 0 & 0 & 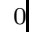 & 0 & 0 & 0 & 0 & 1 & -4 & -2 & 21 & -5 & $5-37$ & 12 & 24 & -5 & -4 & 5702 \\
\hline 0 & 0 & 0 & 0 & 0 & 0 & 0 & 0 & 1 & -4 & -2 & 21 & -5 & $5 \mid-36$ & 10 & 21 & -1 & -1 & 8720 \\
\hline 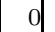 & 0 & 0 & 0 & 0 & 0 & 0 & 0 & 1 & -4 & -2 & 22 & -8 & $8-38$ & 20 & 21 & -9 & -1 & 7479 \\
\hline 0 & 0 & 0 & 0 & 0 & 0 & 0 & 0 & 1 & -3 & -6 & 20 & 13 & $3-44$ & -13 & 34 & 4 & -7 & 471 \\
\hline 0 & 0 & 0 & 0 & 0 & 0 & 0 & 0 & 1 & -3 & -5 & 17 & & $9-31$ & -7 & 18 & 1 & -1 & 58 \\
\hline 0 & o & 0 & 0 & 0 & 0 & 0 & 0 & 1 & -3 & -5 & 17 & & $9-30$ & -8 & 16 & 3 & -1 & 334 \\
\hline 0 & 0 & 0 & & 0 & 0 & 0 & 0 & 1 & -3 & -5 & 18 & & $7 \mid-34$ & -1 & 20 & -3 & -1 & 247 \\
\hline 0 & 0 & 0 & 0 & 0 & 0 & 0 & 0 & 1 & -3 & -5 & 20 & & $3 \mid-41$ & -12 & 25 & -10 & -1 & 64 \\
\hline 0 & 0 & 0 & 0 & 0 & 0 & 0 & 0 & 1 & -2 & -8 & 13 & 25 & $5-26$ & -35 & 14 & 16 & 1 & 07 \\
\hline 0 & & 0 & & 0 & 0 & 0 & 0 & 1 & -2 & -8 & 14 & 23 & $3-30$ & -28 & 18 & 12 & 1 & 88 \\
\hline 0 & & 0 & ( & l & 0 & 0 & . & 1 & -2 & -8 & 15 & 22 & $2-37$ & -23 & 32 & 6 & -5 & 14 \\
\hline 0 & & 0 & 0 & ا & 0 & 0 & . & 1 & -2 & -7 & 12 & 17 & $7-22$ & -15 & 13 & 3 & -1 & 93 \\
\hline 0 & & 0 & & 0 & 0 & 0 & 0 & 1 & -2 & -7 & 13 & 16 & $6-26$ & -13 & 16 & 3 & -2 & 66 \\
\hline 0 & & 0 & 0 & 0 & 0 & 0 & 0 & 1 & -2 & -7 & 13 & 16 & $6-26$ & -13 & 17 & 3 & -3 & 87 \\
\hline 0 & & 0 & 0 & 0 & 0 & 0 & 0 & 1 & -2 & -7 & 14 & 15 & $5-30$ & -10 & 19 & 2 & -1 & 28 \\
\hline 0 & 0 & 0 & 0 & 0 & 0 & 0 & 0 & 1 & -1 & -9 & 7 & 28 & $8 \mid-15$ & $|-34|$ & 9 & 12 & 1 & 6732 \\
\hline 0 & & 0 & & 0 & 0 & 0 & 0 & 1 & -1 & -9 & 7 & 28 & $8-15$ & -34 & 10 & 12 & -1 & 3.95293 \\
\hline 0 & & 0 & & 0 & 0 & & 0 & 1 & -1 & -8 & 7 & 21 & $1-15$ & -20 & 10 & 5 & -1 & 436 \\
\hline 0 & & t & & & 0 & & 0 & & & -9 & & 27 & & -30 & o & & -1 & 257 \\
\hline 0 & & 0 & & & 0 & & & & & 30 & & 56 & 6 & 63 & 28 & 21 & 1 & 316 \\
\hline c & & & & & 0 & & & -5 & & 26 & -22 & -46 & 43 & 31 & -23 & -6 & 1 & 382 \\
\hline c & & ( & & 0 & 0 & & & -5 & & 26 & -21 & -49 & 40 & 42 & -20 & -15 & -1 & $\$ 11$ \\
\hline 0 & & & & إ & 0 & & & -5 & & 22 & -22 & -32 & 35 & 19 & -16 & -4 & 1. & 3. \\
\hline 0 & & 0 & & 0 & 0 & d & 1 & -4 & -3 & 24 & -2 & -49 & 11 & 36 & -8 & -6 & 1 & 74 \\
\hline 0 & & 0 & & 0 & 0 & 0 & & -4 & -3 & 25 & -4 & -53 & 16 & 43 & -8 & -11 & -1 & 653 \\
\hline 0 & & 0 & & 0 & 0 & 0 & 1 & -4 & -3 & 26 & -7 & -56 & 28 & 44 & -19 & -10 & I & 3. \\
\hline 0 & & 0 & & 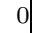 & 0 & 0 & & -4 & -2 & 22 & -8 & -39 & 22 & 23 & -13 & -2 & l & 08 \\
\hline c & & 0 & & 0 & 0 & 0 & 1 & -3 & -7 & 24 & 16 & -67 & $7-12$ & 75 & 0 & -27 & 1 & 291 \\
\hline c & & 0 & & 0 & 0 & $\Omega_{1}$ & & -3 & -6 & 19 & 15 & -40 & $0-20$ & 29 & 11 & -4 & -1 & 10 \\
\hline c & & 0 & & 0 & 0 & 0 & & -3 & -6 & 21 & 11 & -49 & \begin{tabular}{l|l}
9 & -5
\end{tabular} & 42 & -3 & -10 & 2 & 3. \\
\hline c & & 0 & ( & 0 & 0 & 0 & & -1 & -10 & 8 & 36 & -21 & $1 \mid-56$ & 20 & 36 & -5 & -7 & 3. \\
\hline ( & & 0 & 8 & 0 & 0 & إ & & -1 & -10 & 8 & 36 & -21 & $1 \mid-55$ & 20 & 31 & -5 & -3 & 99 \\
\hline ( & & 0 & & 0 & 0 & d & & -1 & -10 & 9 & 35 & -28 & $8 \mid-49$ & 35 & 21 & -15 & & 03 \\
\hline c & & 0 & 0 & 0 & 0 & إ & & -1 & - & 10 & 34 & -34 & $4-43$ & 43 & 12 & -12 & & 301 \\
\hline ( & & 0 & 0 & 0 & 0 & 0 & 1 & 0 & -1 & 0 & 43 & & $1 \mid-70$ & -5 & 41 & 3 & -4 & 766 \\
\hline ( & 0 & 0 & & 0 & 0 & & (1) & 0 & -1 & 0 & 44 & & $0-77$ & 0 & 55 & & -11 & 929 \\
\hline 0 & 0 & 0 & 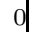 & 0 & 0 & & 1 & 0 & -10 & 0 & 35 & -1 & $1 \mid-5$ & 5 & 25 & -5 & -1 & 3.92140 \\
\hline
\end{tabular}




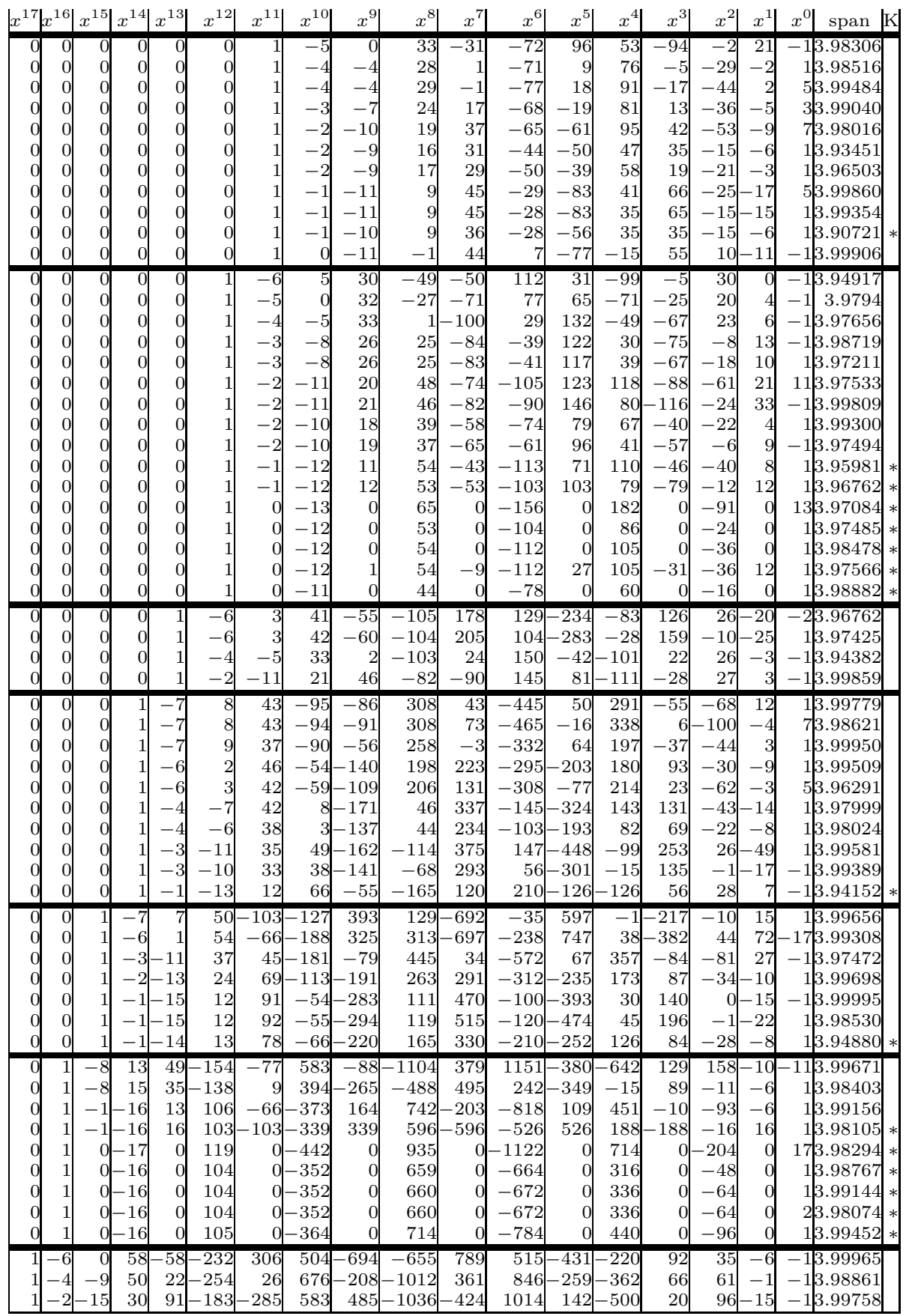




\section{ACKNOWLEDGMENTS}

The computation has been carried out on the ENEA-GRID infrastructure (www.eneagrid.enea.it) and CRESCO HPC system (www.cresco.enea.it). We are particularly thankful to Silvio Migliori and Giovanni Bracco from the FIM Department of ENEA, the Italian National Agency for New Technologies, Energy and the Environment. This work makes use of results produced with the EGEE (http://www.eu-egee.org) grid infrastructure, co-funded by the European Commission (INFSO-RI-222667)

\section{REFERENCES}

1. E. Becker and T. Wörmann, On the trace formula for quadratic forms, Contemp. Math., 155 (1994), 271-291. MR:1260713 (95f:12002)

2. C. Hermite, Remarques sur le théorème de Sturm, C. R. Acad. Sci. Paris, 36 (1853), 32-54.

3. L. Kronecker. Zwei Sätze über Gleichungen mit ganzzahligen Coefficienten, J. Reine Angew. Math., 53 (1857), 173-175.

4. P. Pedersen, M. F. Roy and A. Szpirglas, Counting real zeros in the multivariate case, Progr. Math., 109 (1993), 203-223. MR1230868 (94m:14075)

5. Q. I. Rahman and G. Schmeisser, Analytic Theory of Polynomials, London Mathematical Society New Series, 26, Oxford Univ. Press 2002. MR.1954841 (2004b:30015)

6. J. C. Mason and D. C. Handscomb, Chebyshev polynomials. Chapman \& Hall/CRC, Boca Raton, 2003. MR:1937591(2004h:33001)

7. R. Robinson, Intervals containing infinitely many sets of conjugate algebraic integers, Studies in Mathematical Analysis and Related Topics: Essays in honor of George Pólya, Stanford Univ. Press, 1962, 305-315. MR0144892 (26:2433)

8. R. Robinson, Algebraic equations with span less than 4, Math. Comp., 18 (1964), 547-559. MR 0169374 (29:6624)

9. I. Schur, Uber die Verteilung der Wurzeln bei gewissen algebraischen Gleichungen mit ganzzahligen Koeffizienten, Math. Z., 1 (1918), 377-402. MR.1544303

Dipartimento di Metodi e Modelli Matematici per le Scienze Applicate, Università di Roma "La Sapienza", Via Scarpa 16, I-00161 Roma, Italy

E-mail address: capparelli@dmmm.uniroma1.it

Dipartimento di Metodi e Modelli Matematici per le Scienze Applicate, Università di Roma "La Sapienza", Via Scarpa 16, I-00161 Roma, Italy

E-mail address: alberto.delfra@uniroma1.it

ENEA Fim, Via E. Fermi 45, I-00044 Frascati (RM), Italy

E-mail address: scio@frascati.enea.it 